\title{
Texture formation process of 6063-type aluminium alloy during hot extrusion
}

\author{
Masahiro Araki ${ }^{1, *}$ Kenji Matsuda ${ }^{2}$, Seungwon $\mathrm{Lee}^{2}$, Taiki Tsuchiya ${ }^{2}$, Susumu Ikeno ${ }^{3}$ \\ 1 Development Headquaters, YKK AP Inc., Kurobe-shi, Toyama, 938-8613, Japan \\ 2 Graduate School of Science and Engineering for Research, University of Toyama, Toyama-shi, Toyama, 930-8555 \\ 3 Professor emeritus, University of Toyama, Toyama-shi, Toyama, 930-8555
}

\begin{abstract}
This study investigated the texture formation process of A6063 alloy hot extruded material using electron backscatter diffraction (EBSD) measurements. The cube component that becomes the main orientation during hot extrusion was formed near the bearing inflow in the extrusion chamber and grew with continuous recrystallization. The Goss component with the same ED // $<100>$ relationship as the cube component was not formed in the chamber or the bearing but was formed by discontinuous recrystallization after passing through the bearing. The TD // <111 $>$ orientation component of the surface layer was formed in the bearing and then grew with discontinuous recrystallization. However, because the internal cube and Goss components expanded preferentially, the surface layer TD // <111> components were replaced by the cube and Goss components after passing through the bearing. The cubic texture formation of extruded aluminium alloys is noted to be analogous to the formation of rolling sheet materials, formed by recovery and recrystallization from a plane strain deformation structure. However, for the A6063 alloy extruded under conditions close to industrial production, the cube components were mainly formed by recovery and continuous recrystallization.
\end{abstract}

\section{INTRODUCTION}

The texture of aluminium and aluminium alloys has been investigated for a long time. In the 1920 s, studies reported the [111] orientation structure developed in the drawing direction of an aluminium wire rod and a double fiber structure of [111] and [100] in a round wire [1]. Although there are fewer studies on this topic compared to research on rolled materials, extensive research has been conducted on the relationship between the texture and mechanical properties of extruded $\mathrm{Al}-\mathrm{Mg}$-Si alloys since the 1960s. Research on the recrystallized texture of aluminium alloys gained remarkable attention in the 2000s, [2-3]. Adachi et al. [4] evaluated the effect of zirconium on the texture formation of extruded aluminium and showed that $\mathrm{Al}_{3} \mathrm{Zr}$ promotes dynamic recrystallization. VanGeertruyden et al. [5] described the geometric dynamic recrystallization that occurs during the pressing of a Cr-added 6061 alloy. Niisato et al. [6] described the cross-sectional shape of extruded Al-Zn$\mathrm{Mg}-\mathrm{Cu}$ alloys and the texture and material strength after extrusion. They also described the recovery and recrystallized structures and their crystal orientations. $\mathrm{Wu}$ et al. [7] reported the effect of Si content in extruded $\mathrm{Al}-\mathrm{Si}-\mathrm{Mg}$ alloys on the recovery structure and recrystallization behavior after extrusion.

In this context, we previously investigated the influence of extrusion conditions on the texture of the A6063 alloy under conditions close to industrial production. Our results indicated that the cube component is the main orientation. The abundance ratio of the cube component changes with the extrusion temperature and extrusion speed [8]. Furthermore, the abundance ratio of the cube component changes with the amount of solute elements such as $\mathrm{Mg}$ and $\mathrm{Si}$; however, the main orientation (i.e., the cube orientation) does not change [9].

In recent years, attention has been paid to the extrusion and recrystallized texture of the 6000 series alloys, but only a few studies have reported on the texture forming process during hot extrusion, [10-11]. However, as with production of rolled sheet materials, it is important to know the details of texture formation in the hot extrusion process to improve material properties by texture control.

Thus, we herein report on the details of the microstructure and texture formation process from the extrusion chamber to the bearing part of the hot extruded material of the A6063 alloy.

\section{Experimental Method}

\subsection{Materials and processing}

Table 1 shows the chemical compositions of the alloys employed herein. The material is a commercial A6063 alloy. Billets with a diameter of $152 \mathrm{~mm}$ were prepared by continuous casting using this alloy. The billets were homogenized in an electric furnace and cut further into

\footnotetext{
* Corresponding author: author@e-mail.org
} 
Table 1 chemical composition of specimens

(mass\%)

\begin{tabular}{ccccccccc}
\hline Alloy & $\mathrm{Mg}$ & $\mathrm{Si}$ & $\mathrm{Fe}$ & $\mathrm{Cu}$ & $\mathrm{Mn}$ & $\mathrm{Cr}$ & $\mathrm{Ti}$ & $\mathrm{Al}$ \\
\hline $\mathrm{A} 6063$ & 0.45 & 0.46 & 0.13 & 0.005 & 0.008 & 0.003 & 0.014 & Bal. \\
\hline
\end{tabular}

billets of a 42-mm diameter and 100-mm length. Subsequently, a profile with an H-shape (Fig. 1) was extruded at an extrusion mold temperature of $813 \mathrm{~K}$ and a ram speed of $10 \mathrm{~mm} / \mathrm{s}$. The calculated extrusion ratio was 28.9. After extrusion of the sample for chamber and bearing structure observations, the mold was removed and water-cooled.

The extrusion die has a structure via which the bearing part can be divided, and after the die is cooled, the material remaining in the chamber is taken out. The sample for microstructural observations of the chamber and bearing was prepared based on the position shown in Fig. 2.

\subsection{Microstructure Analysis}

The crystal orientations were observed and measured using field-emission scanning electron microscopy (FESEM, JSM-7001F) coupled with electron backscatter diffraction (EBSD, TSL : Digiview IV OIM detector). OIM 6 was used to analyze the crystal orientation. In this report, a boundary was defined when the angle difference between adjacent measurement points was $2^{\circ}$ or more. In addition, the area surrounded by the boundary with an angle difference of $5^{\circ}$ or more was considered as a crystal grain, and the average crystal

Table 2 Extrusion conditions

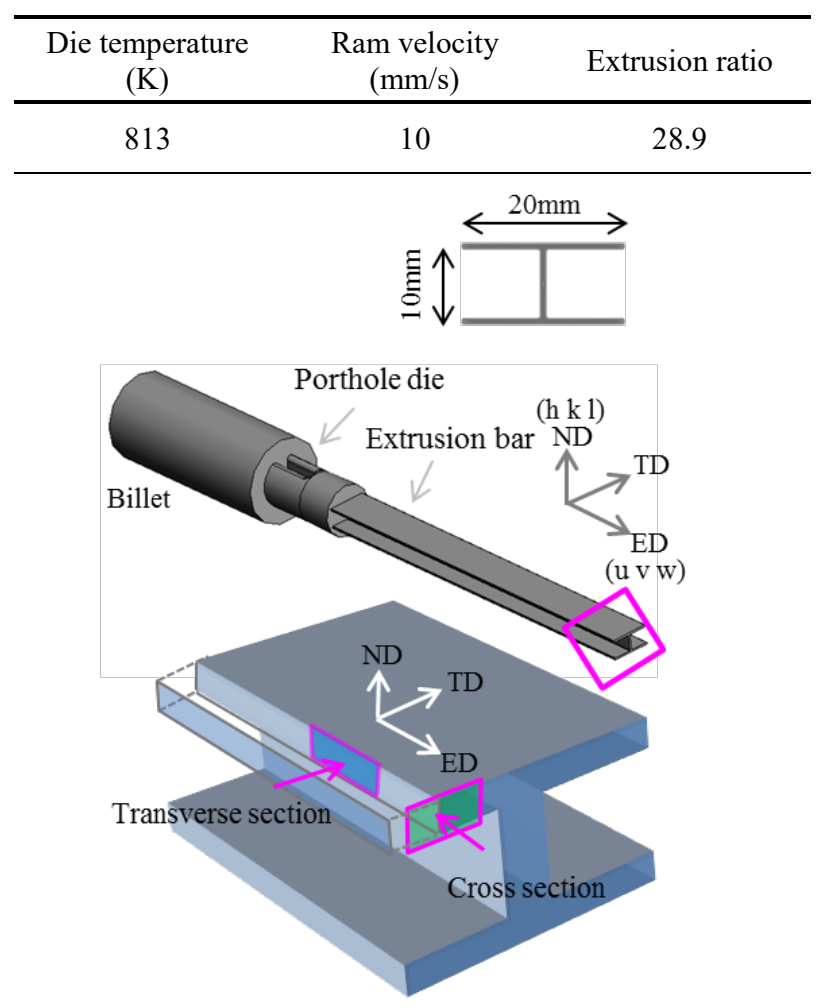

Fig. 1 Schematic of extruded sample. grain size was calculated using the area method. A boundary with an angle difference of $15^{\circ}$ or more from an adjacent crystal grain was defined as a high-angle grain boundary, and a boundary with an angle difference between $2^{\circ}$ and $15^{\circ}$ was defined as a low-angle grain boundary.

The SEM and EBSD samples were prepared by electropolishing at $273 \mathrm{~K}$ and $15 \mathrm{~V}$ for $180 \mathrm{~s}$ using a polishing solution with a perchloric acid:ethanol ratio of 1:9. The structure of the extruded material was observed in the ND / ED plane ( cross section, perpendicular to TD ). A texture component having a plane parallel to the $\mathrm{ED} / \mathrm{TD}$ plane is represented by the index $\{\mathrm{h} \mathrm{k} 1\}$, and the direction parallel to the ED is represented by the index $\langle\mathrm{u} v \mathrm{w}\rangle$. Table 2 shows the extrusion conditions for sample preparation.

Table 3 Specific orientations

\begin{tabular}{|c|c|}
\hline $\begin{array}{l}\text { Orientation } \\
\text { name }\end{array}$ & $\begin{array}{c}\text { Orientation indices } \\
\{\mathrm{h} \mathrm{k} 1\}\langle\mathrm{u} v \mathrm{w}\rangle\end{array}$ \\
\hline Cube & $\left\{\begin{array}{lll}0 & 0 & 1\end{array}\right\}\left\langle\begin{array}{lll}1 & 0 & 0\end{array}\right\rangle$ \\
\hline Goss & $\left\{\begin{array}{lll}0 & 1 & 1\end{array}\right\}\left\langle\begin{array}{lll}1 & 0 & 0\end{array}\right\rangle$ \\
\hline Brass & $\left\{\begin{array}{llll}0 & 1 & 1\end{array}\right\}\left\langle\begin{array}{llll}2 & 1 & 1\end{array}\right\rangle$ \\
\hline TD30Bs & $\left\{\begin{array}{lll}1 & 1 & 2\end{array}\right\}\langle 1110\rangle$ \\
\hline Q & $\left\{\begin{array}{llll}0 & 1 & 3\end{array}\right\}\left\langle\begin{array}{llll}3 & 3 & 1\end{array}\right\rangle$ \\
\hline $\mathrm{S}$ & $\{123\}\langle 634\rangle$ \\
\hline
\end{tabular}

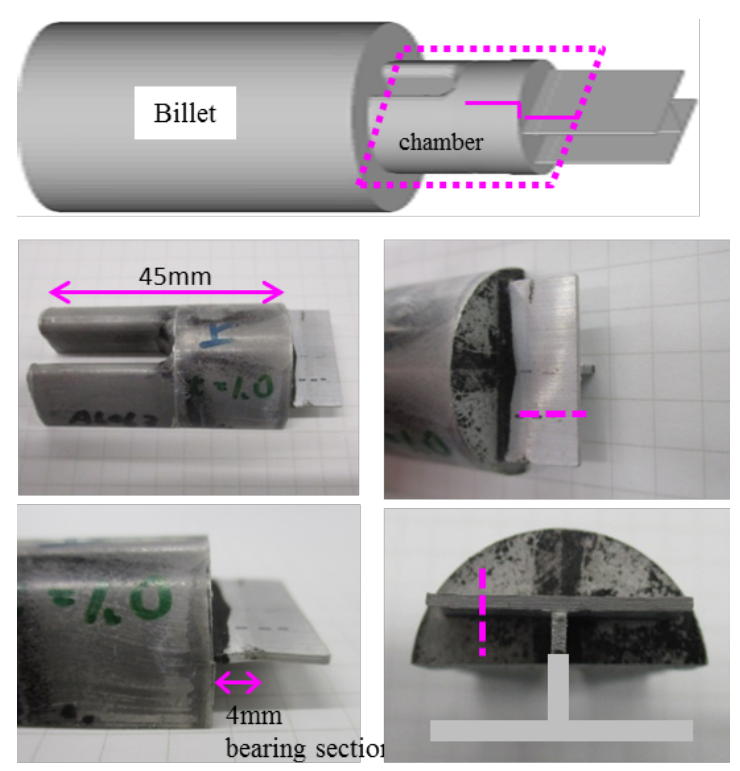

Fig. 2 Sampling locations of extrusion chamber and bearing section 


\subsection{FEM simulation}

Flow simulation was conducted using the FEM software HyperXtrude (Altair Engineering, Inc.)

Deformation during hot extrusion was calculated by the following model.

$$
\begin{gathered}
\sigma=1 / \alpha \sinh ^{-1}(Z / A)^{1 / n} \\
Z=\dot{\varepsilon} \exp (Q / R T)
\end{gathered}
$$

Formura (2) is the Zener-Hollomon parameter, $Q$ is the activation energy, $R$ is the Universal gas constant. $\alpha, A$,

$n, Q$ are material constants these are shown in Table 4 . Die temperature is set to $813 \mathrm{~K}$ and heat transfer coefficient between aluminium and die is $3000 \mathrm{~W} /\left(\mathrm{m}^{2} \mathrm{~K}\right)$. Viscoplastic friction model with a friction coefficient of 0.3 were used at the bearing and chamber section.

Table 4 Material constants used for FEM simulation

\begin{tabular}{cccc}
\hline$\alpha(1 / \mathrm{MPa})$ & $\ln A$ & $\mathrm{n}$ & $Q(\mathrm{~J} / \mathrm{mol})$ \\
\hline 0.04 & 22.5 & 5.385 & 141550 \\
\hline
\end{tabular}

\section{Results and discussions}

\subsection{Microstructure in the extrusion chamber}

To observe changes in the structure and crystal orientation during the hot extrusion process of the A6063 alloy, the structure in the extrusion chamber was monitored. Microstructural observations were performed by cutting the billet remaining in the extrusion chamber along the ED/TD plane. Fig. 3 shows the results of microstructural observations by SEM-EBSP. From the inverse pole figure map (IPF-map) in Fig. 3 (a), the same color is observed to be continuously arranged along the metal flow. The crystal orientation map shown in Fig. 3 (b) indicates that specific crystal orientations such as Brass, TD30Bs, and Q components are arranged along the metal flow. Cube-oriented grains that become the main orientation after extrusion were scarcely present in the chamber, until nearly before the bearing inflow. The ratio of cube grain components in the observation area was approximately $1 \%$.
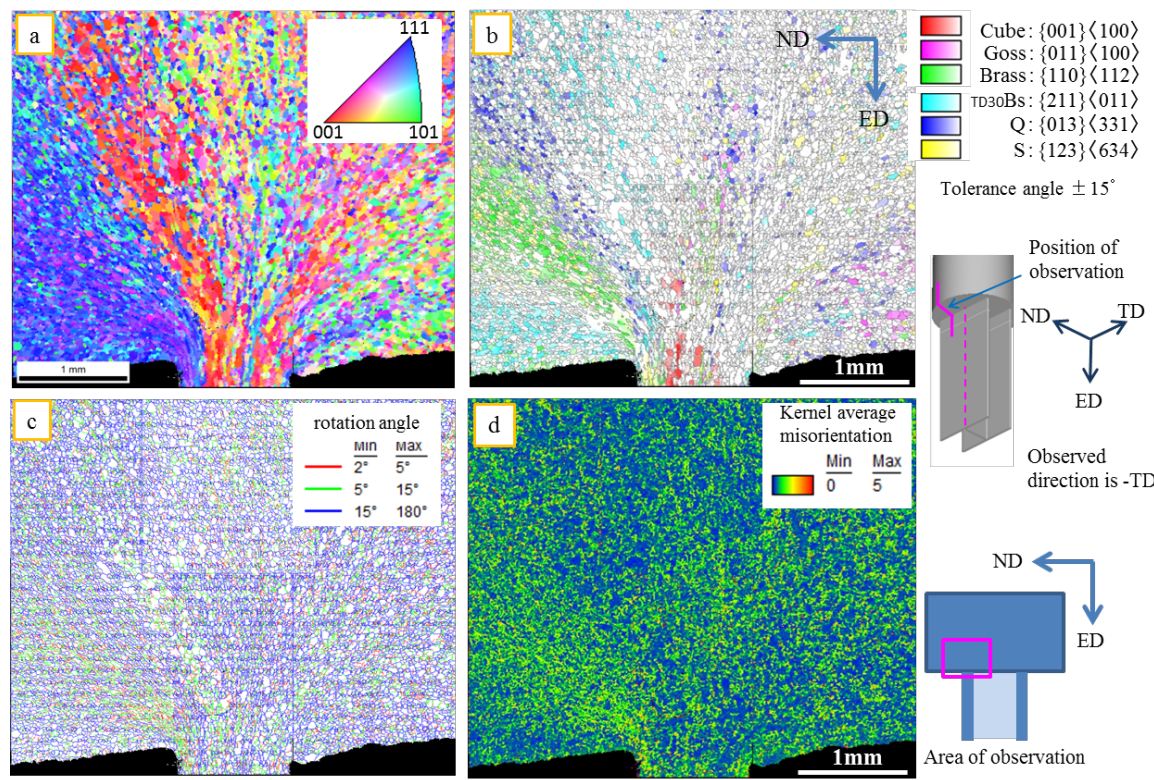

Fig. 3 (a) IPF map, (b) Crystal orientation map, (c) Grain boundary rotation angle image, (d) Kernel average misorientation map on extruded chamber section of A6063 alloy.
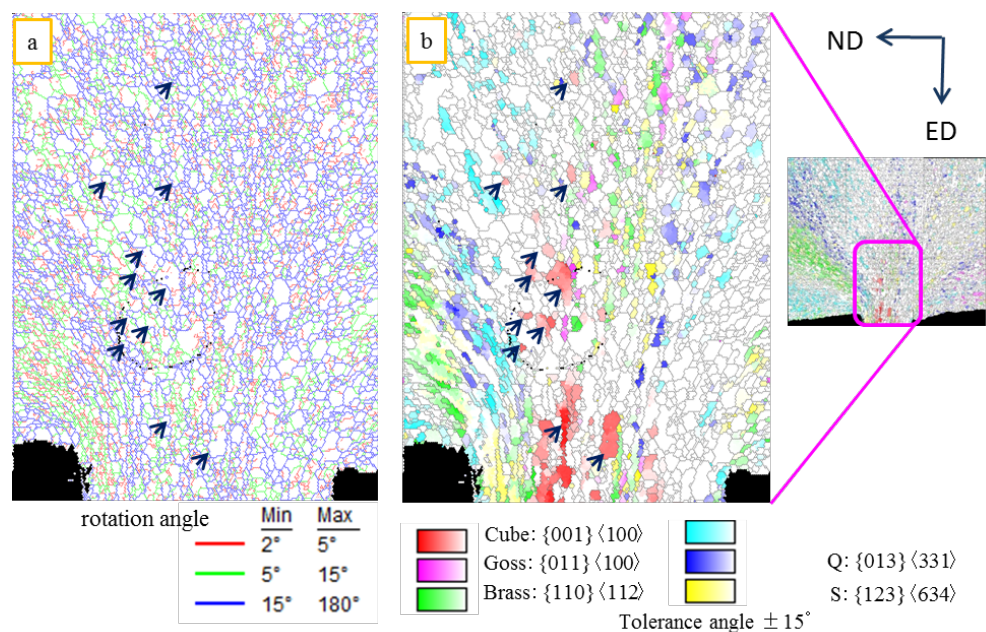

Fig. 4 Magnified image of bearing inlet section on extruded A6063.

(a) Grain boundary rotation angle image and (b) Crystal orientation map. 
Analysis of the grain boundary map shown in Figure 3 (c) showed that about $40 \%$ of the grain boundaries were low angle grain boundaries $\left(<15^{\circ}\right)$. Fig. $3(\mathrm{~d})$ is a Kernel average misorientation map (KAM map) of the same observation area. It can be seen that the green/yellow area is widely distributed in the blue area. The KAM value shows the angle difference from other measurement points in the crystal grain and does not indicate the amount of strain of the material. However it is assumed that the yellow area is the state where distortion remains. As shown in the grain boundary map of Fig. 3(c), many low-angle boundaries existing in the grains were observed. Therefore, the microstructure in the chamber part did not only include recrystallized grains but also consisted of recovered structures. It is noted that the structure formed by the presence of the cube orientation in the chamber was confirmed immediately before the bearing inflow. However, to determine its formation process, the region was expanded and analyzed.

The results are shown in Fig. 4. The crystal orientation map in Fig. 4(b) indicates that many cubic grains (shown in red grains in the figure) exist before and at the bearing inflow. The positions of the cube-oriented grains are also shown. Arrows on the grain boundary map in Fig. 4 (a) indicate the positions where cube-oriented grains exist. These arrows point to grains with low angle grain boundaries with a direction difference of 2 to $15^{\circ}$. And many of the grains were surrounded by low-angle grain boundaries. Cube-oriented grains were not observed in crystal grains surrounded by high-angle grain boundaries observed in other parts of the chamber or in crystal grains that did not contain low-angle grain boundaries. It is thought that cube components not formed by the discontinuous recrystallization but mainly formed by the recovery.

\subsection{Microstructure in the bearing part}

The cube orientation component has been reported to become the main orientation after hot extrusion in Al$\mathrm{Mg}-\mathrm{Si}$ alloys [12]. As a result of observing a crosssection of the sample prepared in this study, the cube component present in an area ratio of approximately $30 \%$ became the main orientation. However, in the chamber shown in Fig. 3, approximately only $1 \%$ of the cube orientation component was present. The average crystal grain size was $52 \mu \mathrm{m}$ in the chamber but increased to $98 \mu \mathrm{m}$ in the extruded profile. This suggests that the cube component increases and expands from the chamber to the passage through the bearing. Therefore, we monitored the microstructure from the chamber to the bearing part and beyond.

Fig. 5 shows the results of microstructural observations of the bearing part from the chamber of the extruded A6063 alloy. The length of the parallel part of the bearing is $4 \mathrm{~mm}$. In order to examine the relationship between the bearing position and the microstructure, the bearing is shown schematically in Fig. 5. From the crystal orientation map, the cube components shown in red in the figure rapidly increase from the bearing inflow. In the bearing part, TD30Bs components are formed on the surface of the extruded.

Fig. 6 shows an enlarged image of the microstructure from the inflow to the bearing. Fig. 6 (a), (b), and (c) are enlarged views of the bearing outflow and Fig. 6 (A) and (B) are the bearing inflows. A comparison of the microstructure of the surface layer between the inflow and outflow of the bearing confirms that the outflow grains are growth rapidly.

The grain boundary map circled areas in Fig. 6 (b) shows that there are few low-angle boundaries appear in the surface of the outflow component.

Looking at the KAM map in Fig. 6 (c), the area inside the profile is displayed in green or yellow. while the surface area surrounded by a circle is displayed in blue.
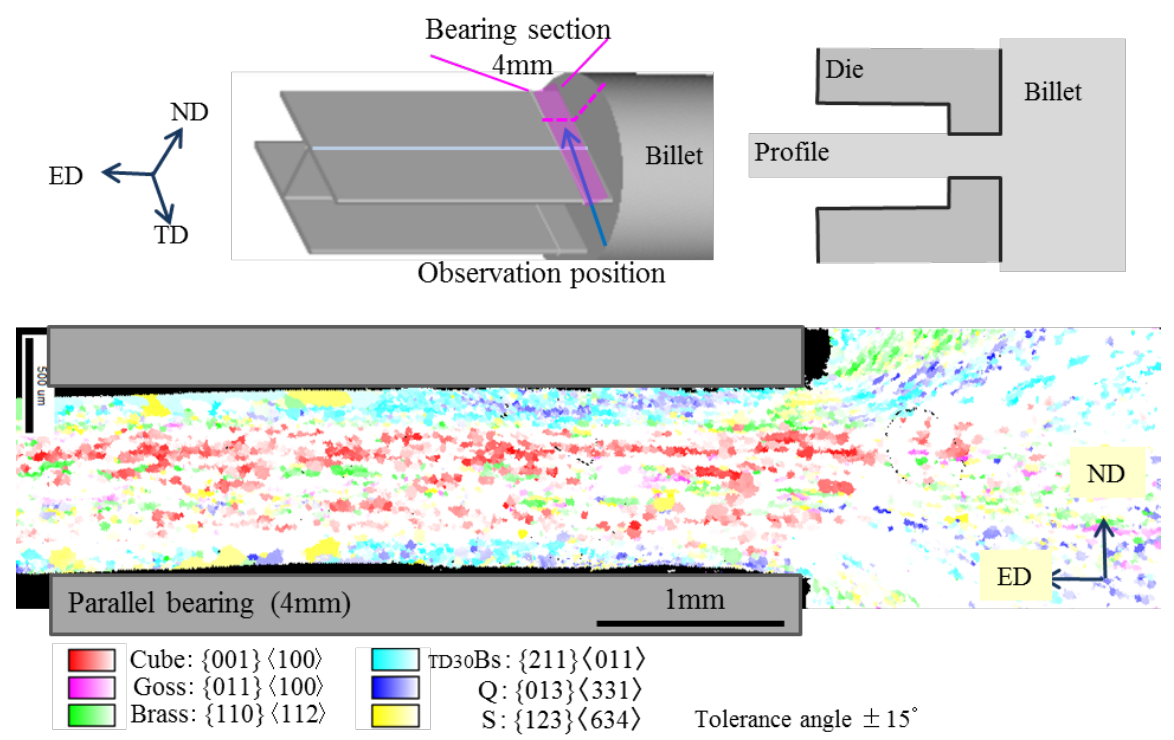

Fig. 5 Crystal orientation map on extrusion bearing part of A6063 alloy. 
The KAM value represents the orientation difference within the same crystal grain. And the surface layer is displayed in blue indicate that the orientation difference within the crystal grain is smaller than that inside crystal grain and close to zero. The orientation difference in the crystal grains does not directly represent the amount of strain, but the crystal grains on the surface layer have no misorientation in the crystal grains, so these are considered to be discontinuous recrystallized grains.

The location of cube grains is shown Fig. 6. The positions of cube grains shown in red in Figs. 6 (a) and (A), these are correspond to the arrows on the grain boundary maps in Figs. 6 (b) and (B). The location of the cube grains is shown on the grain boundary map. These locations overlap with the location where low-angle grain boundaries were observed, as indicated by green and red lines in Fig. 6 (b) and (B). These observations indicate that cube grains are formed in front of the bearing and recovery at the extrusion bearing part. On the grain boundary map, there is no change in the crystal grain size and the grain boundary angle.

In the extruded profile on the bearing part, low-angle grain boundaries were continuously connected. Focusing on the bearing from the chamber, cube orientation grains are formed in this region without significant changes in grain size and orientation. In addition, cube components are formed in a region where many low-angle boundaries exist. So it suggested that cube grains generated by crystal rotation and dynamic recovery accompanying metal flow. The location of the cubic grains is indicated by arrows on the KAM map in Fig. 6 (c); however, the cube components do not appear in the recrystallized region shown in blue in the figure. Therefore, it can be considered that the cube orientation component dose not formed by discontinuous recrystallization. Several phenomena were confirmed to occur simultaneously in the bearing part, such as the formation of the cube component and the recrystallization of the profile surface layer.

\subsection{Comparison with simulation results}

The microstructural observations and crystal orientation analysis in the bearing part indicate that cube components are formed by recovery in the profile. Brass and TD30Bs components are formed at the surface layer and grow in the bearing outflow. To investigate differences in the structure between the interior of the profile and the surface layer and the cause of the microstructure change at the bearing outflow, the metal flow velocity and the shear stress generated in the bearing were calculated by simulation.

Fig. 7 shows a visualized image of the metal flow velocity and shear stress in the bearing and a grain boundary map corresponding to the simulation. Considering the metal flow velocity results in Fig. 7 (a), the material in contact with the bearing at the inflow is a dead metal that hardly moves. The interior material flow velocity is large. The presence of dead metal is inferred

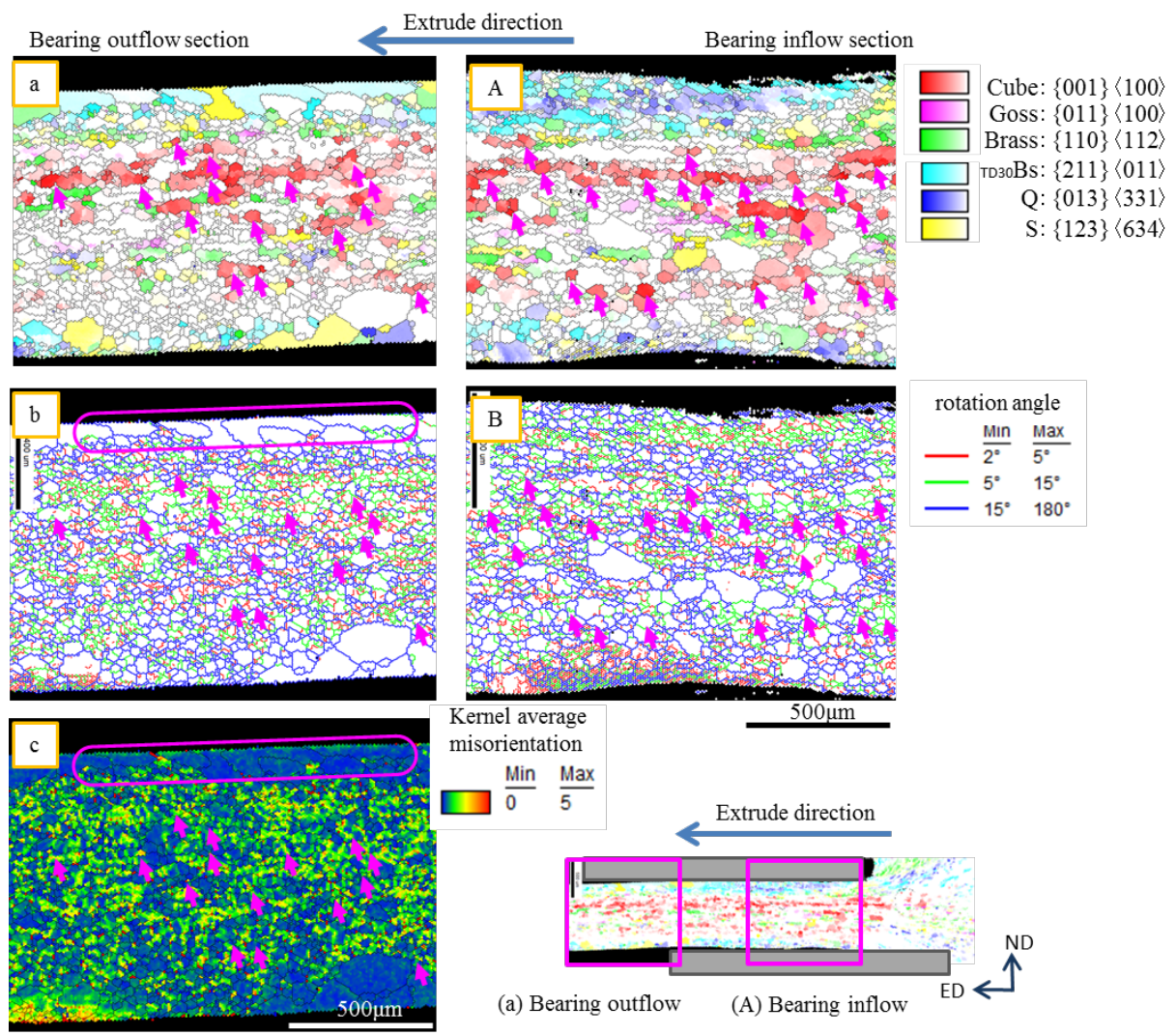

Fig. 6 Crystal orientation map (a), (A). grain boundary rotation angle image (b), (B) and KAM map (c) at bearing section.

(a), (d), and (c) are bearing outlet sections, (A), and (B) are bearing inlet sections 
from the black deposits on the profile of the bearing inflow in Fig. 2. The area with large flow velocity difference overlaps with the area with large shear stress. Consequently, the surface layer shear stress is large up to nearly the middle of the bearing, and the shear stress decreases in the latter half of the bearing. Compared to the microstructural change shown in Fig. 7 (c), the region where recrystallized grains began to appear on the surface of the profile and the part where the shear stress decreased almost coincide. This indicates that the formation of the microstructure in the bearing part is affected by the shear stress and the relaxation of the stress owing to metal flow from the chamber to the bearing and the difference in flow velocity between the profile surface and the interior of the bearing.

\subsection{Microstructure and texture of extruded profile}

The microstructural observations of the bearing showed that the cube component was formed at the bearing inflow of the chamber and increased in the bearing. However, the ratio of the cube component was approximately $20 \%$ in the bearing, which was smaller than that of the extruded material. Table 5 shows the main component abundance ratios in the chamber, bearing, and extruded material. After passing through the bearing, the profile extruded by $1.5 \mathrm{~m}$ had a cube orientation component of $30 \%$ and a Goss orientation component of $9.1 \%$; these values were greater than those of the bearing. The ratio of Brass, TD30Bs, and S components decreased. In particular, the Goss orientation component was scarcely present in the chamber and the bearing; however, the abundance ratio was the second largest (after the cube orientation component) in the extruded profile after passing through the bearing. Fig. 8 shows a crystal orientation map and a grain boundary map of the ND/ED plane (transverse section) of the extruded material after passing through the bearing. Show in the crystal orientation map in Fig. (a), the proportions of the cube and Goss components are higher than that in the bearing, and these components extend to the surface layer of the profile. The location of the cube orientation grains in the crystal orientation map is indicated by an arrow, and the location of the Goss orientation grains is indicated by a gray triangle in the crystal grain map in Fig. 8 (a) and (b). Considering the grain boundary map, the region with many low-angle boundaries are represented by red and green lines, and crystal grains surrounded by high-angle grain boundaries are observed. It can be seen that many cube components are present in the crystal grains surrounded by the lowangle boundaries or including the low-angle boundaries. This tendency is similar to the bearing part. In contrast, many Goss-oriented grains were present in grains surrounded by high-angle grain boundaries. Goss components were scarcely present in the chamber and

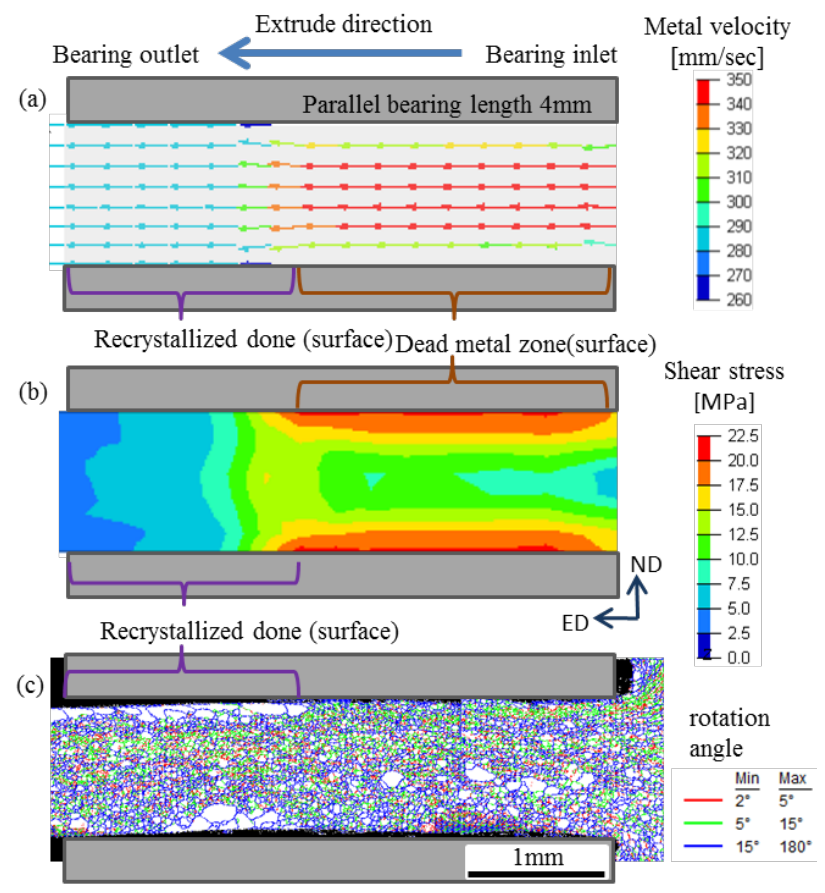

Fig. 7 Extrusion simulation results of (a) metal flow velocity, (b) shear stress at bearing section, and (c) grain boundary rotation angle image at the extruded bearing section

the bearing; however, they are abundant in the extruded profiles. Because Goss grains are crystal grains surrounded by high-angle grain boundaries, they were considered to be newly formed and grown by discontinuous recrystallization after passing through the bearing.

The texture formation process of the aluminium hot extruded material was examined by analyzing the microstructure and crystal orientation of the chamber, the bearing, and the extruded material after passing through the bearing. Fig. 9 shows a schematic of the formation of the cube orientation (i.e., the main orientation after extrusion) and the TD // <111> orientation component of the surface layer.

Table 5 Area fractions of specific direction $(\%)$

\begin{tabular}{cccc}
\hline Orientation name & chamber & Bearing & profile \\
\hline Cube & 1.3 & 20.3 & 30.0 \\
Goss & 1.9 & 2.1 & 9.1 \\
Brass & 7.8 & 6.5 & 0.7 \\
TD30Bs & 10.8 & 12.8 & 2.6 \\
Q & 8.9 & 6.4 & 5.4 \\
S & 6.1 & 8.1 & 4.2 \\
\hline Grain size $(\mu \mathrm{m})$ & 52 & 62 & 98 \\
\hline
\end{tabular}




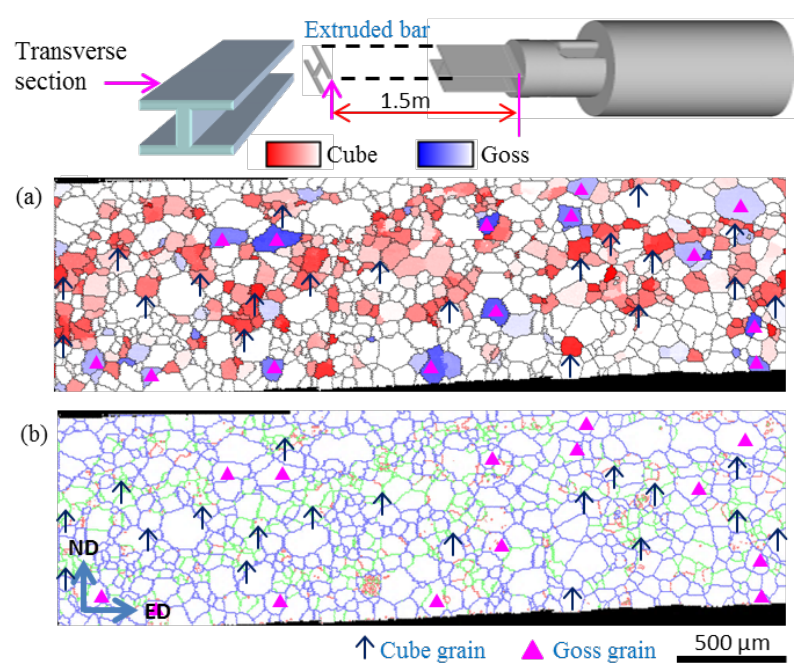

Fig. 8 (a) Crystal orientation map and (b) Grain boundary map of transverse section on extruded profile

\section{Summary}

In order to confirm the formation process of cubeoriented grains in an extruded A6063 alloy, the microstructure in the bearing part from the extrusion chamber was observed. Consequently, cube components were confirmed to be generated before the bearing inflow in the chamber and increased in the bearing part. The cube orientation component existed in structures surrounded by low-angle boundaries and in crystal grains containing low-angle boundaries. From these results, it was concluded that the cube component was primarily formed by recovery.

It has been reported that formation process of cube components of extruded aluminium alloys is similar to that of rolling sheet materials. This process has been explained by recovery and recrystallization from the plane strain deformation structure [13]. In this study, we could not confirm the processed structure due to the plane strain in the chamber and bearing and the cube orientation component recrystallized from the processed structure.

However, cube grains appeared before the bearing inflow, suggesting that they were formed by crystal rotation due to metal flow in the chamber. In the hot extrusion process of the A6063 alloy, recrystallization proceeds from the surface layer of the profile when passing through the bearing part, and crystal grains with a TD // <111> orientation relationship, such as Brass, S, and TD30Bs, are formed. However, most recrystallized grains formed inside the extrusion die showed either Goss or random orientations. This may be due to the differences in shear stress and metal flow velocity between the surface and the interior of the extruded profile.

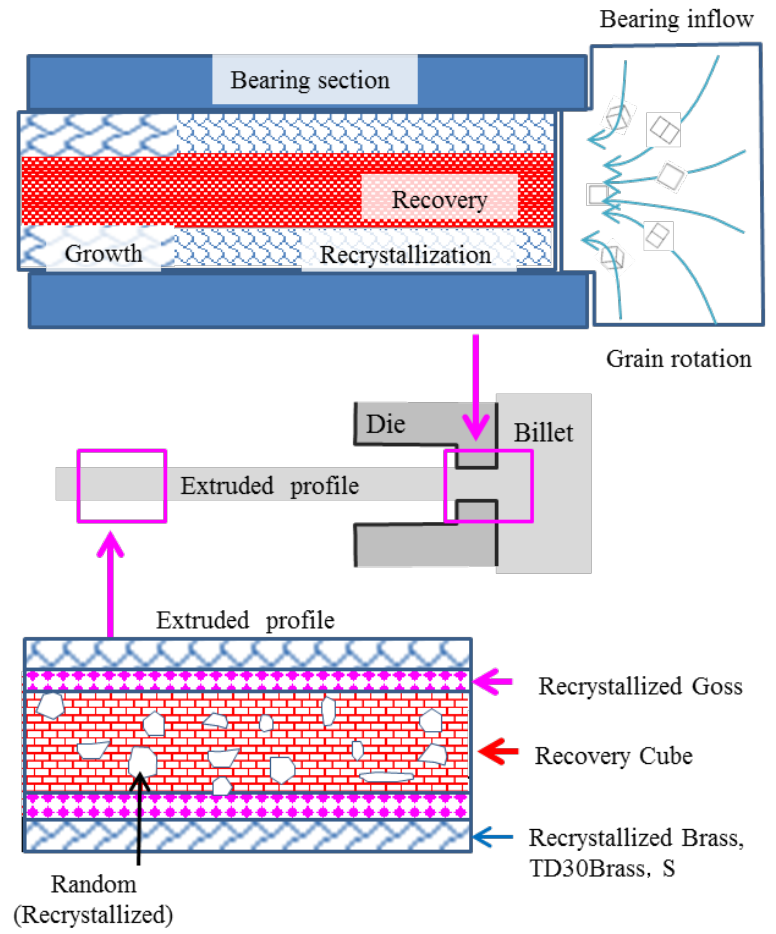

Fig. 9 Schematic of texture formation process of hot extruded A6063.

Acknowledgements : The authors would like to thank Hideaki Nakagawa from YKK Machinery and Engineering Group, support on the extrusion simulation.

\section{References}

1. A. Ono. Men. Coll. Engeg.Kyoshu, 3 (1923/25),195.

2. K. Dies and P. Winciers. Z.Metallkd, 57 (1966), 141, 227.

3. H. Inoue. J. JILM 52 (2002) 617-620

4. H. Adachi, K. Osamura, K. Kikuchi and J. Kusui. Mater Trans JIM 46 (2005) 211-214

5. W. H. VanGeertruyden, W. Z. Misiolek, and P. T. Wang. J Matrl Sci 40 (2005) 3861-3863

6. Y. Niisato, T. Minoda and H. Yoshida. J. JILM 63 (2013) 218-222

7. Y. Wu, H. Liao, J. Yang and K. Zhou. J. Mater. Sci. Tehnol. 30 (2014) 1271-1277

8. M. Araki, K. Matsuda, S. Lee, T. Tsuchiya and S. Ikeno. J. JILM 69 (2019) 327-331

9. M. Araki and K. Matsuda. Mater Trans 61 (2020) 104-110

10. C. Zhang, C. Wang, Q. Zhang, G. Zhao, and L. Chen. J. Mater. Proc. Tech. 270 (2019) 323-334

11. Yu, J. Q., Zhao, G. Q., Zhang, C. S., Chen, L., Mater. Sci. Eng. A 682 (2017) 679-690

12. K. Ihara, T. Shikama and K. Morita. Kobe Steel engineering reports 62 (2012) 43-47

13. J. Baumgarten, W. Bunk, and K. Lücke: Z.Metallkd., 72 (1981), 162-168 